\title{
Erratum: Ko et al. (2021)
}

In the article Ko J, Deprez D, Shaw K, Alcorn J, Hadjistavropoulos T, Tomczak C, Foulds H, Chilibeck PD. Stretching is superior to brisk walking for reducing blood pressure in people with high-normal blood pressure or stage I hypertension. $J$ Phys Act Health. 2021;18(1):21-28. https://doi.org/10.1123/jpah.2020-0365, the clinical trial registration number was incorrectly listed in the text and acknowledgments as NCT02391779; the correct registration number is NCT03947996. The online version of this article has been corrected. The authors regret this error. 